\title{
Coded-Reference Ultra-Wideband Systems
}

\author{
Sinan Gezici \\ Department of Electrical and Electronics Engineering \\ Bilkent University, Bilkent, Ankara 06800, Turkey \\ gezicidee.bilkent.edu.tr
}

\begin{abstract}
Transmitted-reference (TR) and frequency-shifted reference (FSR) ultra-wideband (UWB) systems employ pairs of reference and data signals, which are shifted in the time and frequency domains, respectively, to facilitate low-to-medium data rate communications without the need for complex channel estimation and template signal generation. On the other hand, the recently proposed coded-reference $(C R)$ UWB systems provide orthogonalization of the reference and data signals in the code domain, which has advantages in terms of performance and/or implementation complexity. In this paper, CR UWB systems are investigated. First, it is shown that a CR UWB system can be considered as a generalized non-coherent pulse-position modulated system. Then, an optimal receiver according to the Bayes decision rule is derived for CR UWB systems. In addition, the asymptotic optimality properties of the conventional CR UWB receivers are investigated. Finally, simulation results are presented to compare the performance of the optimal and conventional CR UWB receivers.

Index Terms- Ultra-wideband (UWB), transmitted-reference
\end{abstract} (TR), frequency-shifted reference (FSR), coded-reference (CR), Bayes decision rule.

\section{INTRODUCTION}

In addition to high-speed data transmission [1] and accurate position estimation [2], pulse-based ultra-wideband (UWB) signals can also facilitate low-to-medium rate communications with low-power and low-cost transceivers. In order to realize such low-power/cost implementations, one can consider transmitted-reference (TR) UWB systems, which transmit a pair of signals that are time delayed versions of each other for each information symbol [3], [4]. A TR UWB receiver can use one of those signals as a reference ("template") signal for the other one (called the data signal) to estimate the transmitted information. The main advantages of TR UWB receivers are that there is no need to estimate individual channel coefficients and template signals, which is quite challenging for UWB systems, and that the receiver can be operated based on symbolrate or frame-rate samples. However, the main disadvantage of TR UWB receivers is related to the need for an analog delay line to perform data demodulation [3], [5].

In order to realize the advantages of TR UWB systems without the need for an analog delay line, slightly frequency-shifted reference (FSR) UWB systems are proposed, which employ data and reference pulses that are shifted in the frequencydomain instead of the time domain [5]. One limitation of FSR UWB systems is that the orthogonality between the data and reference signals cannot be maintained at the receiver for high data rate systems [6].

Recently, coded-reference (CR) $\mathrm{UWB}^{1}$ systems are pro-

${ }^{1}$ In [6], this system is called "code-orthogonalized transmitted-reference UWB", whereas [8] calls it as "code-multiplexed UWB transmittedreference". In order to provide terminology unification and to be consistent with the original terms "transmitted-reference UWB" and "frequency-shifted reference UWB", the system is called "coded-reference UWB" in this paper. posed, which perform orthogonalization of reference and data signals by means of polarity codes [6]-[8]. Similar to FSR UWB, CR UWB systems do not need analog delay lines, and they also have better bit-error-rate (BER) performance than FSR UWB and TR UWB systems [8]. In addition, they do not have the data rate limitation that FSR UWB systems experience [6].

In this paper, CR UWB systems are investigated, and optimal and suboptimal CR UWB receivers are studied. First, a generic signal model for TR, FSR, and CR UWB systems is introduced (Section II), and it is shown that a CR UWB system can be modeled as a generalized non-coherent pulseposition modulated system (Section III). Then, an optimal receiver according to the Bayes decision rule is derived for CR-UWB systems. In addition, the optimality properties of the conventional CR UWB receivers are investigated, and it is shown that the conventional receiver provides a low-cost solution that is close-to-optimal for practical system settings (Section IV). Finally, simulation results are presented and concluding remarks are made (Section $\mathrm{V}$ ).

\section{Signal Model}

First, a generic signal structure is defined, which covers TR, FSR and CR UWB signals as special cases. The signal corresponding to the 0th symbol is expressed as

$$
\begin{aligned}
s_{0}(t)=\sqrt{\frac{E_{\mathrm{s}}}{2 N_{\mathrm{f}}}} & \sum_{j=0}^{N_{\mathrm{f}}-1}\left[a_{j} \omega\left(t-j T_{\mathrm{f}}-c_{j} T_{\mathrm{c}}\right)\right. \\
& \left.+b_{0} a_{j} \omega\left(t-j T_{\mathrm{f}}-c_{j} T_{\mathrm{c}}-T_{\mathrm{d}}\right) x(t)\right]
\end{aligned}
$$

for $t \in\left[0, T_{\mathrm{s}}\right]$, where $T_{\mathrm{s}}, T_{\mathrm{f}}$ and $T_{\mathrm{c}}$ are, respectively, the symbol, frame and chip intervals, $N_{\mathrm{f}}$ is the number of frames per symbol, $E_{\mathrm{s}}$ is the symbol energy, $a_{j} \in\{-1,+1\}$ represents a polarity randomization code, which is useful for spectral shaping [9], $c_{j}$ is the time-hopping (TH) code, $\omega(t)$ is the UWB pulse with unit energy, and $b_{0} \in\{-1,+1\}$ is the binary information symbol. The other signal parameters are specified for each system as follows:

- For TR systems, $T_{\mathrm{d}}$ represents the time delay between the reference and data pulses in each frame, and $x(t)=1 \forall t$.

- For FSR systems, $T_{\mathrm{d}}=0$ and $x(t)=\sqrt{2} \cos \left(2 \pi f_{0} t\right)$, which provides a slight frequency shift to the data pulses [5].

- For CR systems, $T_{\mathrm{d}}=0$ and $x(t)$ is given by

$$
x(t)=\sum_{j=0}^{N_{\mathrm{f}}-1} \tilde{d}_{j} p\left(t-j T_{\mathrm{f}}\right),
$$

where $p(t)=1$ for $t \in\left[0, T_{\mathrm{f}}\right]$ and $p(t)=0$ otherwise, and $\tilde{d}_{j} \in\{-1,+1\}$ is the $j$ th element of the code that 
provides orthogonalization of the data pulses and the reference pulses at the receiver [8].

Note that TR systems provide orthogonalization of the data and reference signals by separating them in the time-domain, whereas FSR systems facilitate separation via a shift in the frequency-domain. On the other hand, the approaches in [6] and [8] propose a separation in the code domain, which has significant advantages over the previous techniques in terms of performance and/or implementation complexity. $[8]$

From (2), (1) can be expressed for CR UWB systems as ${ }^{2}$

$$
s(t)=\sqrt{\frac{E_{\mathrm{s}}}{2 N_{\mathrm{f}}}} \sum_{j=0}^{N_{\mathrm{f}}-1}\left(a_{j}+b d_{j}\right) \omega\left(t-j T_{\mathrm{f}}-c_{j} T_{\mathrm{c}}\right),
$$

where $d_{j} \doteq a_{j} \tilde{d}_{j}$. Note that $\left(a_{j}+b d_{j}\right)$ takes a value from the set $\{-2,0,+2\}$.

Assume that the signal in (3) passes through a multipath channel with the channel impulse response $h(t)=$ $\sum_{l=1}^{L} \alpha_{l} \delta\left(t-\tau_{l}\right)$, where $\alpha_{l}$ and $\tau_{l}$ represent, respectively, the channel coefficient and delay of the $l$ th path. Then, the received signal can be expressed from (3) as

$$
r(t)=\sqrt{\frac{E_{\mathrm{s}}}{2 N_{\mathrm{f}}}} \sum_{j=0}^{N_{\mathrm{f}}-1}\left(a_{j}+b d_{j}\right) \tilde{\omega}\left(t-j T_{\mathrm{f}}-c_{j} T_{\mathrm{c}}\right)+n(t),
$$

for $t \in\left[0, T_{\mathrm{s}}\right]$, where $\tilde{\omega}(t)=\sum_{l=1}^{L} \alpha_{l} \omega\left(t-\tau_{l}\right)$ and $n(t)$ is zero mean Gaussian noise with a flat spectral density of $\sigma^{2}$ over the system bandwidth. It is assumed that the frame interval is sufficiently long and the TH codes are selected in such a way that there occurs no inter-frame interference (IFI) [6]. Note that due to the no IFI assumption, signal demodulation can be performed symbol-by-symbol without loss of optimality. Hence, only one symbol is considered in (4). In addition, since a single-user scenario is considered, no $\mathrm{TH}$ codes are considered, i.e., $c_{j}=0 \forall j$, without loss of generality.

\section{RECEIVER StRUCTURES}

In order to estimate the information symbol $b$ from the received signal in (4), the orthogonality between the reference and data pulses is used [6], [8]. Namely, the information symbol is estimated as

$$
\hat{b}=\operatorname{sgn}\left\{\int_{0}^{T_{\mathrm{s}}} r^{2}(t) x(t) \mathrm{d} t\right\},
$$

where $\operatorname{sgn}\{\cdot\}$ represents the sign operator. The detector in (5) can be implemented as shown in the first receiver structure in Fig. 1.

From (2), (5) can also be expressed as

$$
\hat{b}=\operatorname{sgn}\left\{\sum_{j=0}^{N_{\mathrm{f}}-1} \tilde{d}_{j} \int_{j T_{\mathrm{f}}}^{(j+1) T_{\mathrm{f}}} r^{2}(t) \mathrm{d} t\right\},
$$

which suggests another detector implementation based on frame-rate samples [8], as shown in the second structure in Fig. 1.

\footnotetext{
${ }^{2}$ The symbol index 0 is dropped for convenience.
}

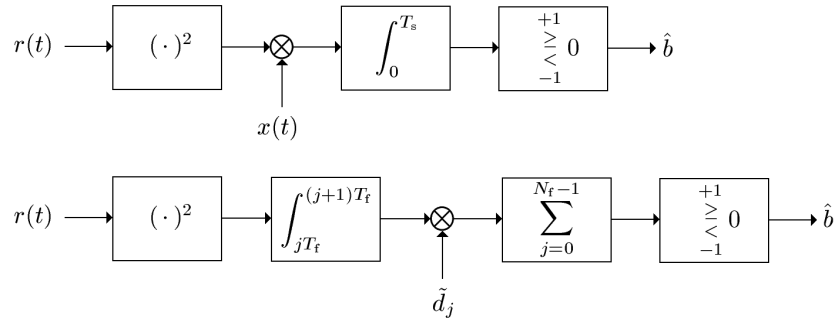

Fig. 1. Receivers for CR UWB signals. The first receiver employs symbolrate sampling, whereas the second one uses frame-rate sampling.

Although both receivers in Fig. 1 can be considered in the framework of code orthogonalized/multiplexed signals [6], [8], it is also possible to consider the current system as a "generalized" pulse-position modulation (PPM) system. To that end, let $\mathcal{S}$ and $\mathcal{S}$ represent the sets of frame indices for which $\tilde{d}_{j}=1$ and $\tilde{d}_{j}=-1$, respectively; i.e.,

$$
\begin{aligned}
& \mathcal{S}=\left\{j \in \mathcal{F} \mid \tilde{d}_{j}=1\right\} \\
& \overline{\mathcal{S}}=\left\{j \in \mathcal{F} \mid \tilde{d}_{j}=-1\right\}
\end{aligned}
$$

where $\mathcal{F}=\left\{0,1, \ldots, N_{\mathrm{f}}-1\right\}$ is the set of frame indices. Note that $\mathcal{S} \cup \overline{\mathcal{S}}=\mathcal{F}$. In addition, both sets include $N_{\mathrm{f}} / 2$ indices for orthogonalization purposes [8]; i.e., $|\mathcal{S}|=|\overline{\mathcal{S}}|=N_{\mathrm{f}} / 2$.

Note that for $b=1$, the pulses are transmitted in the frames indexed by $\mathcal{S}$ and no pulses are transmitted in the frames indexed by $\overline{\mathcal{S}}$ (c.f. (3)). Similarly, for $b=-1$, the pulses are transmitted in the frames indexed by $\overline{\mathcal{S}}$ and no pulses are transmitted in the frames indexed by $\mathcal{S}$.

Also it is observed from (6) that comparing the sum of $N_{\mathrm{f}}$ outputs against zero is equivalent to comparing the sum of the positive outputs against the absolute value of the sum of the negative outputs. Therefore, (6) can be expressed, using (7) and (8), as

$$
\sum_{j \in \mathcal{S}} \int_{j T_{\mathrm{f}}}^{(j+1) T_{\mathrm{f}}} r^{2}(t) \mathrm{d} t \underset{\substack{\hat{b}=-1 \\ \gtrless}}{\gtrless} \sum_{j \in \mathcal{S}} \int_{j T_{\mathrm{f}}}^{(j+1) T_{\mathrm{f}}} r^{2}(t) \mathrm{d} t,
$$

which is mainly a non-coherent binary PPM detector. However, unlike conventional PPM systems [10], the signals employed for the binary symbols are not always time-shifted versions of each other, as each signal consists of a number of pulses in different frames of the UWB symbol.

\section{ON THE Optimality OF CR UWB ReCEIVERS}

In order to investigate the optimality of the CR UWB receivers studied in the previous section, we first derive the optimal receiver that estimates the information symbol according to the Bayesian approach [11].

Let $y_{j}=\int_{j T_{\mathrm{f}}}^{(j+1) T_{\mathrm{f}}} r^{2}(t) \mathrm{d} t, j=0,1, \ldots, N_{\mathrm{f}}-1$, represent the set of energy samples obtained from different frames. Then, from (4), (7) and (8), the optimal receiver design problem can be modeled as the following binary hypothesis 
testing problem:

$$
\begin{aligned}
& \mathcal{H}_{0}: y_{j}= \begin{cases}\int_{0}^{T_{\mathrm{f}}} n_{j}^{2}(t) \mathrm{d} t, & j \in \mathcal{S} \\
\int_{0}^{T_{\mathrm{f}}}\left[\omega_{j}(t)+n_{j}(t)\right]^{2} \mathrm{~d} t, & j \in \overline{\mathcal{S}}\end{cases} \\
& \mathcal{H}_{1}: y_{j}= \begin{cases}\int_{0}^{T_{\mathrm{f}}}\left[\omega_{j}(t)+n_{j}(t)\right]^{2} \mathrm{~d} t, & j \in \mathcal{S} \\
\int_{0}^{T_{\mathrm{f}}} n_{j}^{2}(t) \mathrm{d} t, & j \in \overline{\mathcal{S}}\end{cases}
\end{aligned}
$$

where $\mathcal{H}_{0}$ and $\mathcal{H}_{1}$ represent the $b=-1$ and $b=1$ hypotheses, respectively, $\omega_{j}(t) \doteq \sqrt{\frac{2 E_{\mathrm{s}}}{N_{\mathrm{f}}}} a_{j} \tilde{\omega}(t)$, and $n_{j}(t) \doteq n\left(t+j T_{\mathrm{f}}\right)$.

Since $n(t)$ is zero mean Gaussian noise with a flat spectral density of $\sigma^{2}$ over the system bandwidth, the energy samples can be shown to be distributed as central and non-central chisquare random variables [12]. Therefore, (10) can be expressed as

$$
\begin{aligned}
& \mathcal{H}_{0}: y_{j} \sim \begin{cases}\chi_{M}^{2}(0), & j \in \mathcal{S} \\
\chi_{M}^{2}(\theta), & j \in \overline{\mathcal{S}}\end{cases} \\
& \mathcal{H}_{1}: y_{j} \sim \begin{cases}\chi_{M}^{2}(\theta), & j \in \mathcal{S} \\
\chi_{M}^{2}(0), & j \in \overline{\mathcal{S}}\end{cases}
\end{aligned}
$$

where $M$ is the approximate dimensionality of the signal space, which is obtained from the time-bandwidth product [12], $\theta$ is the signal energy (in the absence of noise), which can be obtained as $\theta=2 E_{\mathrm{s}} E_{\omega} / N_{\mathrm{f}}$, with $E_{\omega}=\int_{-\infty}^{\infty} \tilde{\omega}^{2}(t) \mathrm{d} t$, and $\chi_{M}^{2}(\theta)$ denotes a non-central chi-square distribution with $M$ degrees of freedom and a non-centrality parameter of $\theta$. Clearly, $\chi_{M}^{2}(\theta)$ reduces to a central chi-square distribution with $M$ degrees of freedom for $\theta=0$. For the model in (11), it is assumed that the noise components are independent for energy samples from different frames ${ }^{3}$.

From (11), the optimal receiver can be obtained as in the following proposition.

Proposition 1: For equiprobable information symbols and a uniform cost assignment ${ }^{4}$, the Bayes decision rule for estimating the information symbol $b$ is given by

$$
\prod_{j \in \mathcal{S}} y_{j}^{\frac{1}{2}-\frac{M}{4}} \mathrm{I}_{\frac{M}{2}-1}\left(\frac{\sqrt{\theta y_{j}}}{\sigma^{2}}\right)_{\substack{\hat{b}=-1 \\ \stackrel{b}{<}=+1}}^{\gtrless} \prod_{j \in \overline{\mathcal{S}}} y_{j}^{\frac{1}{2}-\frac{M}{4}} \mathrm{I}_{\frac{M}{2}-1}\left(\frac{\sqrt{\theta y_{j}}}{\sigma^{2}}\right),
$$

where $\mathrm{I}_{\nu}(x)$ for $x \geq 0$ is the $\nu$ th order modified Bessel function of the first kind.

Proof: Please see Appendix A.

Note that the Bayes rule in (12) is also the minimum probability-of-error decision scheme for the given problem due to the assumption of uniform cost assignment [11].

Comparison of (9) and (12) reveals that the conventional receiver in (9) has lower computational complexity than the optimal one since it directly adds up the signal energies in different frames. In addition, the optimal receiver requires the knowledge of $\theta$, which is not readily available in practice. Therefore, the performance of the optimal receiver can be considered to provide a lower bound on that of the conventional

\footnotetext{
${ }^{3}$ This is approximately true in practice since the frame interval is commonly much larger than the inverse of the bandwidth.

${ }^{4} \mathrm{~A}$ uniform cost assignment (UCA) associates each error with the same cost, and assumes no cost for correct decisions [11]. In this case, the two types of errors are "to decide -1 when $b=+1$ " and "to decide +1 when $b=-1 "$.
}

receiver.

Before comparing the performance of the conventional receiver with that of the optimal receiver derived in this section, the asymptotic optimality of the conventional approach will be established in the following. To that end, the following result is obtained first.

Lemma 1: If $M$ is an even number, the optimal receiver in (12) can be expressed as

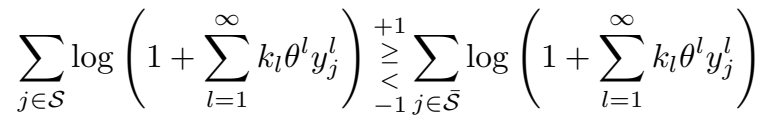

where

$$
k_{l} \doteq\left[\left(2 \sigma^{2}\right)^{2 l} l !\left(\frac{M}{2}\right) \cdots\left(\frac{M}{2}+l-1\right)\right]^{-1}
$$

for $l=1,2, \ldots$

Proof: Please see Appendix B.

The main implication of Lemma 1 regarding the asymptotic optimality of the conventional receiver follows from the observation that for large $M$ values, the logarithm terms in (13) converge to $\theta y_{j} /\left(2 M \sigma^{4}\right)$; hence the test reduces to $(9)$. In other words, if the chi-square random variables representing the signal energies in different frames have large degrees of freedom, then the conventional receiver performs very closely to the optimal one. Note that the degrees of freedom parameter is determined by the product of the bandwidth and the observation interval [12]. Therefore, as the integration interval over which the energy is calculated (in this case, the frame interval, $T_{\mathrm{f}}$ ) increases, $M$ also increases. Note that in practice, the integration can be performed over intervals that are smaller than the frame interval in order to collect less noise and increase the signal-to-noise ratio (SNR) [13]. Therefore, the performance of the conventional receiver should be investigated for various $M$ values in order to determine how close it gets to the optimal receiver in various scenarios, which is studied in the next section.

\section{Simulation Results AND CONCLUSIONS}

In this section, performance of the conventional receiver in (9) is compared with that of the optimal receiver in (12) for various system parameters.

For the first set of simulations, the number of frames, $N_{\mathrm{f}}$, is equal to $10, \sigma^{2}$ is set to unity, and $\theta=10$. In order to investigate the performance of the receivers for various degrees of freedom, BERs are obtained for various $M$ values. For each $M$, the frame interval and/or the bandwidth are adjusted to provide the desired $M$ and no IFI exists in any of the scenarios. Fig. 2 illustrates the BERs of the two receivers. Although the optimal receiver performs better than the conventional one for small $M$, the performance difference is not significant, and the receivers have almost the same performance for $M \geq 8$.

The same simulations are also performed when $N_{\mathrm{f}}=4$ and $\theta=25$, and the results are shown on the same plot in Fig. 2. As in the previous scenario, the conventional receiver performs very closely to the optimal one. In addition, lower BERs are observed compared to the previous scenario.

It follows from both the simulations and the theoretical analysis that the conventional receiver converges to the optimal receiver for sufficiently large $M$ values. Since $M$ is determined 


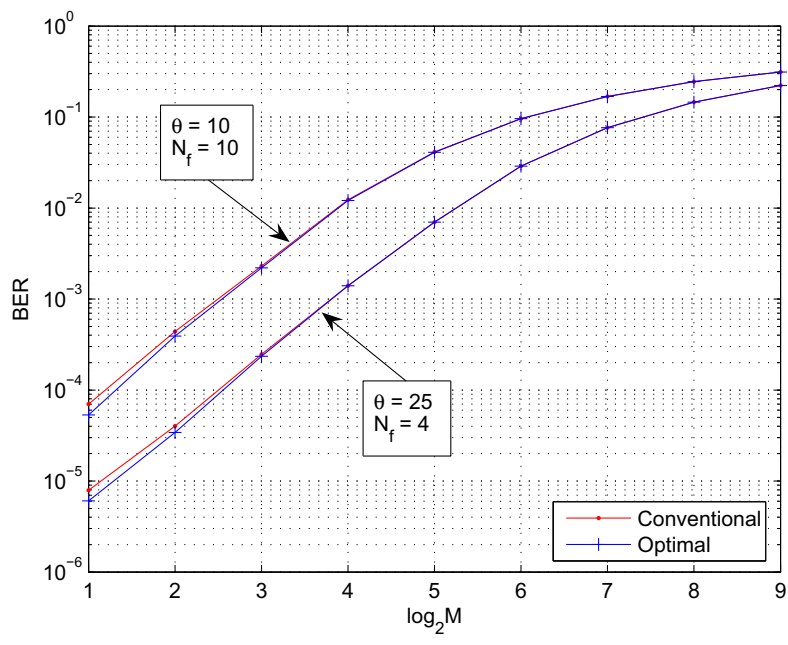

Fig. 2. BER versus $M$ for the conventional and the optimal receivers.

by the multiplication of the signal bandwidth and the integration interval for the frames, larger integration intervals make sure that the conventional receiver performs very closely to the optimal one. In practice, UWB channels commonly have large delay spreads, hence the integration interval cannot be made very small compared to the pulse width. Therefore, in practical cases, $M$ is not expected to be very small, and the conventional receiver has almost the optimal performance. Also note that the conventional receiver has lower computational complexity than the optimal one and it makes almost no assumptions about the signal parameters. Hence, the conventional receiver seems to be a natural choice for demodulating CR UWB signals for the considered system settings.

\section{APPENDIX}

\section{A. Proof of Proposition 1}

For equiprobable information symbols and uniform cost assignment, the Bayes decision rule is given by [11]

$$
L(\mathbf{y})=\frac{p_{1}(\mathbf{y})}{p_{0}(\mathbf{y})} \underset{\substack{\hat{b}=+1 \\ \hat{b}=-1}}{<} 1
$$

where $\mathbf{y}=\left[\begin{array}{ll}y_{0} & y_{1} \cdots y_{N_{\mathrm{f}}-1}\end{array}\right]$ and $p_{i}(\mathbf{y})$ is the conditional probability density function of $\mathbf{y}$ given that the hypothesis $\mathcal{H}_{i}$ is true $(i=0,1)$.

From the independent noise components assumption, $p_{1}(\mathbf{y})$ can be obtained, using (11), as

$$
\begin{aligned}
p_{1}(\mathbf{y}) & =\prod_{j \in \mathcal{S}} \frac{1}{2 \sigma^{2}}\left(\frac{y_{j}}{\theta}\right)^{\frac{M}{4}-\frac{1}{2}} e^{-\frac{\left(\theta+y_{j}\right)}{2 \sigma^{2}}} \mathrm{I}_{\frac{M}{2}-1}\left(\frac{\sqrt{\theta y_{j}}}{\sigma^{2}}\right) \\
& \times \prod_{j \in \overline{\mathcal{S}}} \frac{y_{j}^{\frac{M}{2}}-1}{\sigma^{M} 2^{-\frac{y_{j}}{2 \sigma^{2}}} \Gamma(M / 2)},
\end{aligned}
$$

where $\Gamma(\cdot)$ is the Gamma function [10] and

$$
\mathrm{I}_{\nu}(x)=\sum_{l=0}^{\infty} \frac{(x / 2)^{\nu+2 l}}{l ! \Gamma(\nu+l+1)}
$$

for $x \geq 0$ is the $\nu$-th order modified Bessel function of the first kind.

For $p_{0}(\mathbf{y})$, the expression in (16) can be used by replacing $\mathcal{S}$ and $\mathcal{S}$. Then, (15) can be shown to be equal to (12) after some manipulation.

\section{B. Proof of Lemma 1}

From (17), the decision rule in (12) can be expressed as

$$
\prod_{j \in \mathcal{S}} y_{j}^{\frac{1}{2}-\frac{M}{4}} f_{\theta}\left(y_{j}\right) \stackrel{\hat{b}=+1}{\underset{\hat{b}}{=}=-1} \underset{j \in \overline{\mathcal{S}}}{\gtrless} y_{j}^{\frac{1}{2}-\frac{M}{4}} f_{\theta}\left(y_{j}\right)
$$

where

$$
f_{\theta}\left(y_{j}\right)=\sum_{l=0}^{\infty} \frac{\left(\theta y_{j}\right)^{\frac{M}{4}-\frac{1}{2}+l}}{\left(2 \sigma^{2}\right)^{\frac{M}{2}-1+2 l} l ! \Gamma(M / 2+l)} .
$$

From the facts that $|\mathcal{S}|=|\overline{\mathcal{S}}|=N_{\mathrm{f}} / 2$ and $\Gamma(M / 2+l)=$ $(M / 2+l-1)$ ! (since $M / 2$ is an integer), (18) and (19) can be simplified to ${ }^{5}$

$$
\prod_{j \in \mathcal{S}} \sum_{l=0}^{\infty} k_{l} \theta^{l} y_{j}^{l} \underset{\substack{\hat{b}=+1 \\ \gtrless}}{\gtrless} \prod_{j \in \overline{\mathcal{S}}} \sum_{l=0}^{\infty} k_{l} \theta^{l} y_{j}^{l}
$$

where $k_{l}$ is given by (14) for $l=0,1,2, \ldots$ Then, (13) can be obtained by taking the logarithm of both sides in (20) and using the fact that $k_{0}=1$.

\section{REFERENCES}

[1] H. Arslan, Z. N. Chen, and M.-G. Di Benedetto, (editors) Ultra Wideband Wireless Communications, Hoboken: Wiley-Interscience, Oct. 2006.

[2] S. Gezici, Z. Tian, G. B. Giannakis, H. Kobayashi, A. F. Molisch, H. V. Poor and Z. Sahinoglu, "Localization via ultra-wideband radios," IEEE Signal Processing Magazine, vol. 22, issue 4, pp. 70-84, July 2005.

[3] H. Hoctor and H. Tomlinson, "Delay-hopped transmitted-reference RF communications," Proc. IEEE Conference on Ultra-Wideband Systems and Technologies, pp. 265-270, Baltimore, MD, May 2002.

[4] J. Choi and W. Stark, "Performance of ultra-wideband communication with suboptimal receivers in multipath channels," IEEE Journal on Selected Areas in Communications, vol. 20, no. 12, pp. 1754-1766, Dec. 2002

[5] D. L. Goeckel and Q. Zhang, "Slightly frequency-shifted reference ultrawideband (UWB) radio," IEEE Transactions on Communications, vol. 55, no. 3, pp. 508-519, March 2007.

[6] J. Zhang, H.-Y. Hu, L.-K. Liu, and T.-F. Li, "Code-orthogonalized transmitted-reference ultra-wideband (UWB) wireless communication system," International Conference on Wireless Communications, Networking and Mobile Computing, pp. 528-532, Sept. 21-25, 2007.

[7] J. Zhang, H-Y Hu, and Z-Y Zhang, "Timing acquisition for codeorthogonalized transmitted-reference ultra-wideband (UWB) wireless communication system," IEEE International Workshop on RadioFrequency Integration Technology, pp. 50-53, Dec. 9-11, 2007.

[8] A. A. D'Amico and U. Mengali, "Code-multiplexed UWB transmittedreference radio," IEEE Transactions on Communications, to appear.

[9] Y.-P. Nakache and A. F. Molisch, "Spectral shape of UWB signals influence of modulation format, multiple access scheme and pulse shape," Proc. IEEE Vehicular Technology Conference, vol. 4, pp. 25102514, Jeju, Korea, Apr. 2003.

[10] J. G. Proakis, Digital Communications, 4th ed., New York: McGrawHill, 2001.

[11] H. V. Poor, An Introduction to Signal Detection and Estimation, New York: Springer-Verlag, 1994

[12] P. A. Humblet and M. Azizoğlu, "On the bit error rate of lightwave systems with optical amplifiers," Journal of Lightwave Technology, vol. 9, issue 11, pp. 1576-1582, Nov. 1991.

[13] Z. Tian, and B. M. Sadler, "Weighted energy detection of ultra-wideband signals," Proc. IEEE Signal Processing Workshop on Advances in Wireless Communications, New York, NY, pp. 158-162, June 6-8, 2005.

\footnotetext{
${ }^{5}$ The details are omitted due to the space limitation.
} 